\title{
INVESTIGATION OF CENTRIFUGAL PUMP CHARACTERISTICS
}

\author{
Edgars Repsa, Eriks Kronbergs \\ Latvia University of Life Sciences and Technologies, Latvia \\ edgars.repsa@1lu.lv, eriks.kronbergs@1lu.lv
}

\begin{abstract}
A pump is generally used to induce flow and raise the pressure of a liquid. Centrifugal pumps are a category of dynamic pumps. The working principle of centrifugal pumps involves imparting energy to the liquid by means of a centrifugal force developed by the rotation of an impeller that has several blades or vanes. Education on centrifugal pump systems is important for future engineers, working with agricultural, forest and road construction machinery. For engineering education particularly is needed virtual presentation of laboratory work, especially in centrifugal pump performance determination. On the basis of the experimental test bench obtained centrifugal pump head - capacity and pipeline system resistance curve analyses, it was determined that the curves have a parabolic shape. Difference between the experimentally and analytically obtained centrifugal pump head capacity curve values are les then $5.7 \%$. Analytical model of the pipeline system resistance curve individual for each student lets change the shape of the curve in order to obtain different operating point values. The aim of the present investigation is to improve online engineering education in fluid mechanics, which is used instead of faceto-face learning. By giving students individual coefficients for the parabolic network characteristic equation, they graphically obtain different operating points for the centrifugal pump. This allows students to understand the main method of control of a centrifugal pump - by changing the resistance of the network. The analytical equations of the pump head - capacity characteristic and pipeline system resistance curve allow to model centrifugal pump series and parallel application more conveniently.
\end{abstract}

Keywords: fluid mechanics, education, centrifugal pump, modelling.

\section{Introduction}

Centrifugal pump systems play an important role in the modern world. These systems are used in water supply networks by providing a huge amount of treated and raw water for industrial and commercial users $[1 ; 2]$.

The nowadays research is dedicated to getting pump characteristics for domestic application [3], or performance evaluation of a centrifugal pump variations [4] under different operating conditions. Studies of the geometric parameters of a centrifugal pump impeller results state that their modifications can have a significant impact [5] on its performance. Investigations show that the introduction of air into the water changes [6-8] the pump characteristic curve. Experiences with incorporating the testing of small water pumps in an engineering technology curriculum have shown that it is a versatile and economical tool for learning valuable techniques [9] in experimental testing.

The aim of the present investigation is to improve online engineering education in fluid mechanics, which is used instead of face-to-face learning. The most important and difficult is virtual presentation of laboratory work, especially in centrifugal pump performance determination. For this reason, centrifugal pump head - capacity and pipeline system resistance curves have been obtained experimentally as during usual laboratory work in the face-to-face learning process. On the basis of the experimental test bench obtained centrifugal pump head - capacity and pipeline system resistance curve analyses, it was determined that the curves have a parabolic shape. The obtained analytical equations of the pump head - capacity and pipeline system resistance curves let improve student online teaching to pump adjustment by obtaining different operating points by changing the resistance of the pipeline characteristics.

The analytical equations of the pump head - capacity characteristic and pipeline system resistance curve alow to model centrifugal pump series and parallel application more conveniently. The series and parallel application of pumps offer the following advantages: possibility of improving the Life Cycle Cost (LCC), use of existing equipment including possibility of using equipment lying redundant in the warehouse, better way to cope up with fluctuating demands in the flow. Together with the pipeline system resistance characteristic new operating point values are obtained. By changing the resistance coefficient of the pipeline individually for each student there was an opportunity to assign an individual graphoanalytical work in the virtual study of the centrifugal pump. 


\section{Materials and methods}

One of the basic characteristics of a pump operation is its change in the head $\mathrm{H}$ depending on the pump capacity Q. The total head of a pump is the amount of specific energy that the pump gives to the liquid being transported, expressed in meters of liquid column. Experimental measurements were performed on the existing laboratory test bench. The hydraulic scheme of the laboratory test bench is shown in Fig. 1.

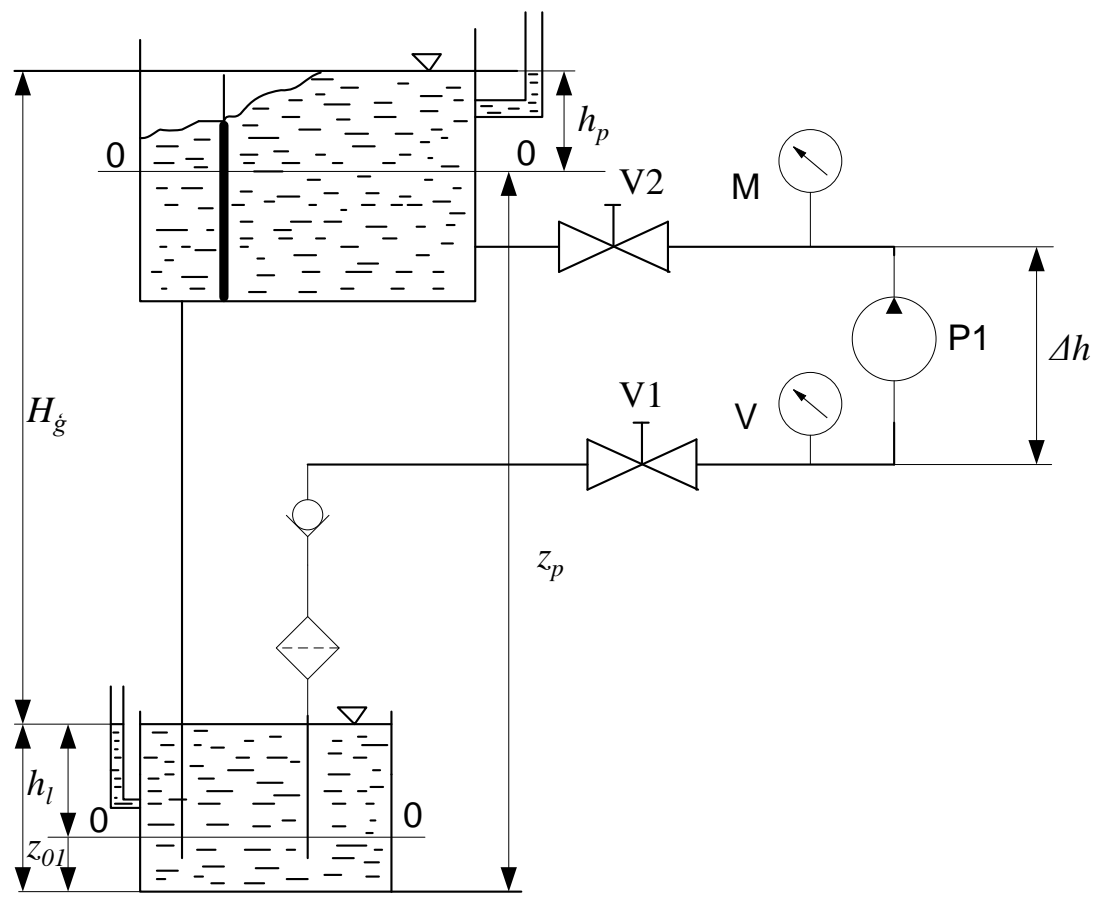

Fig. 1. Test bench scheme

During the experiments the constant values of $z_{01}=0.298 \mathrm{~m}, z_{p}=1.285 \mathrm{~m}$ and $\Delta h=0.31 \mathrm{~m}$ were measured. The electric motor speed $\mathrm{n}=2850 \mathrm{rpm}$. The pump flow rate with the valve V2 was controlled. The pressure reading $p_{m}(M)$ and vacuum gauge reading $p_{v}(V)$ were recorded. The height of the level above the overturning threshold $h_{p}$ and the level in the suction tank $h_{l}$ were read and recorded for 6 different pump flow values. According to the reading, $\mathrm{p}_{\mathrm{m}}$ and $\mathrm{p}_{\mathrm{v}}$ values of the head of the pump $\mathrm{H}$ are calculated by the expression (1).

$$
H=\frac{p_{m}}{\gamma}+\frac{p_{v}}{\gamma}+\Delta h+\frac{v_{s p}^{2}-v_{s}^{2}}{2 g},
$$

were $\quad p_{m}-$ pressure reading, $\mathrm{Pa}$;

$p_{v}$ - vacuum gauge reading, $\mathrm{Pa}$;

$\Delta h$ - height difference between the manometer and vacuum meter connection points, $\mathrm{m}$;

$v_{s p}$ - average speed of the liquid in the pressure line at the connection

point of the manometer, $\mathrm{m} \cdot \mathrm{s}^{-1}$;

$v_{s}$ - average speed of the liquid in the suction line at the connection point of the vacuum meter, $\mathrm{m} \cdot \mathrm{s}^{-1}$;

$\gamma$ - volumetric density, $\mathrm{N} \cdot \mathrm{m}^{-3}$

$g-$ acceleration of gravity, $\mathrm{m} \cdot \mathrm{s}^{-2}$.

The graphical representation of the function $H=H(Q)$ is called the main characteristic of the pump and is found experimentally at a constant drive shaft speed $\mathrm{n}$ for different openings of the valve V2.

For the network or pipeline curve calculation equation (2) was used.

$$
H_{0}=H_{\dot{g}}+a Q^{2},
$$

were $\quad H_{\dot{g}}-$ geodetic lifting head, m; 
$a$ - reduced resistance factor for the pipeline, $s^{2} \cdot m^{-5}$;

$Q$ - pump flow rate, $\mathrm{m}^{3} \cdot \mathrm{s}^{-1}$, if $Q$ is measured $1 \cdot \mathrm{s}^{-1}$, then $a\left[\mathrm{~s}^{2} \cdot \mathrm{m}^{-5} \cdot 10^{-6}\right]$.

The pipeline characteristic is constructed according to equation (2), by placing the experimentally determined pump performance average values from 7 measurements. Value of coefficient "a" is found according to (2) for fully open valve V2, when to the operating point $D$ correspond $H_{0}=H$, and the maximum value $Q$.

\section{Results and discussion}

Experimentally obtained and theoretically determined head curves are shown in Fig. 2.



Fig. 2. Experimental and calculated curves

By constructing the characteristic of the pump and the network in the same coordinate system, the so-called working point $D$ is obtained. When working with a given network, the pump capacity and the developed head is determined by the $D$ coordinates.

The main characteristic of the centrifugal pump $H=H(Q)$ is experimentally obtained and catalogued by manufacturers as a falling curve. As the line $H=H(Q)$ is very similar to a parabola, we constructed the main characteristic of the pump corresponding to equation of parabola $H_{t}=22.8-1.94 Q^{2}$. The numbers in this equation are obtained from coordinates of the working point $D$ and the initial value $A$, then $H=22.8$ and $Q=0$. The pump head value difference of experimentally obtained characteristic with the measurement accuracy $\pm 0.17 \mathrm{~m}$ (confidence level $99.0 \%$ ) and that, described by parabolic equation, is less than $5.7 \%$ for $Q=0.81 \mathrm{~s}^{-1}$. Therefore, they can be considered similar and the parabolic equation can be used as the main characteristic of the pump.

This innovation allows students to virtually experiment with the pump and network characteristics, obtaining different operating points and the corresponding head $H$ and flow rate $Q$. The analytical parabolic main characteristic of the pump and also different parabolic pipeline equation (2) individually for each student let obtain also different values of the working point and head $H$, depending on the pump capacity $Q$. For this aim to each student constant " $a$ " in equation (2) was given individually. The main characteristic of the pump was given by parabolic equation range (see Table 1). The parabolic equation of the network or pipeline for fully open valve V2, expressed by the range in $H_{0}$ column.

If we choose for individual student option No. 2 with $a=20$ from Table 1 , then we get new characteristic of the network, expressed by the range in $H_{0}^{2}$ column. The parabolic equation is $H_{0}^{2}=0.65+20 Q^{2}$ for this option. 
Main characteristic of the pump

\begin{tabular}{|c|c|c|c|c|c|}
\hline No. & $\boldsymbol{a}$ & $\boldsymbol{Q}, \mathbf{l}^{-\mathbf{1}}$ & $\boldsymbol{H}, \mathbf{m}$ & $\boldsymbol{H}_{\mathbf{0}}, \mathbf{m}$ & $\boldsymbol{H}_{\mathbf{0}}^{\mathbf{2}} \mathbf{\mathbf { m }}$ \\
\hline 1 & 10 & 0 & 22.8 & 0.65 & 0.65 \\
\hline 2 & 20 & 0.2 & 22.7 & 0.92 & 1.45 \\
\hline 3 & 30 & 0.4 & 22.5 & 1.73 & 3.85 \\
\hline 4 & 40 & 0.6 & 22.1 & 3.08 & 7.85 \\
\hline 5 & 50 & 0.8 & 21.6 & 4.97 & 13.45 \\
\hline 6 & 60 & 1 & 20.9 & 7.4 & 20.65 \\
\hline 7 & 70 & 1.2 & 20 & 10.37 & 29.45 \\
\hline 8 & 80 & 1.4 & 19 & 13.88 & 39.85 \\
\hline 9 & 90 & 1.6 & 17.8 & 17.93 & 51.85 \\
\hline 10 & 100 & 1.8 & 16.5 & 22.52 & 65.45 \\
\hline
\end{tabular}

As the result for individual student option No. 2 three characteristics - the pump main head characteristic and two pipeline network characteristics are obtained.

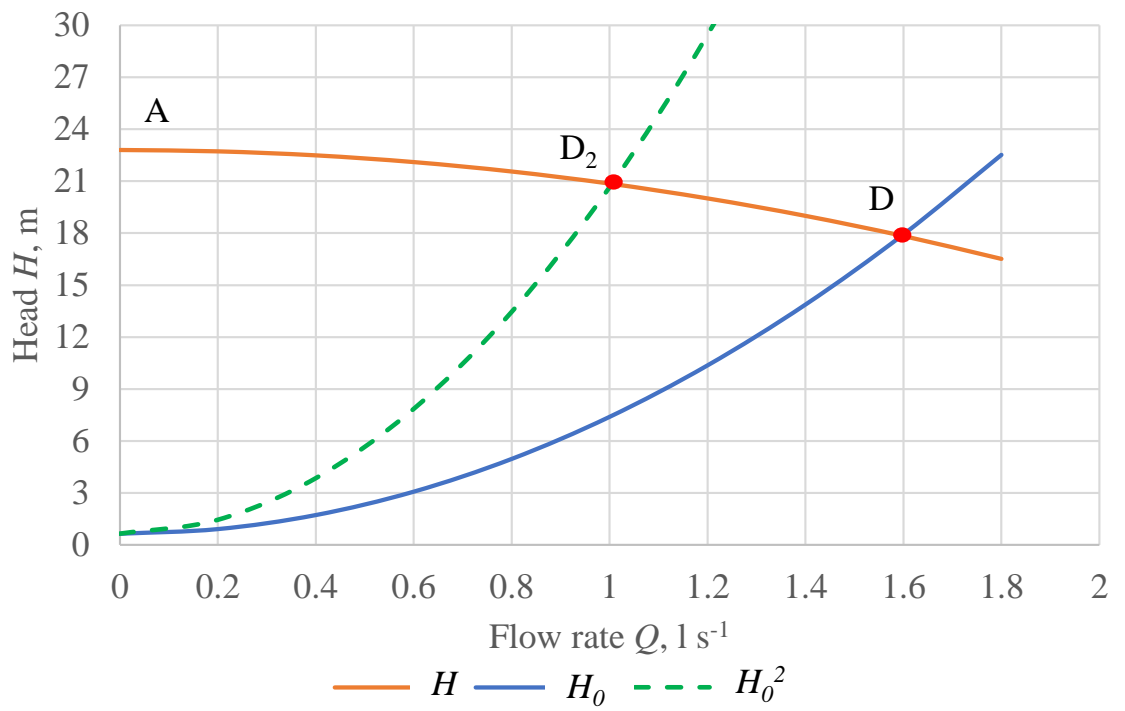

Fig. 3. Pump and pipeline network characteristics

The new pipeline characteristic constructed by students gives on crossing with the main head characteristic the working point $D_{2}$. The coordinates of the point $D_{2}$ determine the pump head $21 \mathrm{~m}$ at the flow rate $1 \mathrm{l} \mathrm{s}^{-1}$ instead of the head $18 \mathrm{~m}$ and the flow rate $1.6 \mathrm{l} \mathrm{s}^{-1}$ for the working point $D$ with fully open valve V2. The analytical parabolic main characteristic of the pump and also different individual parabolic equations of the pipeline network equation (2) for each student let obtain understanding of the method of adjusting the pump parameters. Important is the fact that each student obtains by constructing a graph individual operating working point of the pump. Online virtual investigation of the centrifugal pump control by changing the resistance of the pipeline network used instead of laboratory work on site allows students to obtain individual results and pump control practices remotely.

For two centrifugal pump series and parallel application modeling it is necessary to use the main pump characteristic parameters in graph form together with the pipeline network characteristic. Parallel application can be obtained, using the single pump head curve point head values together with doubled flow rate values (see Fig. 4). Two pump series application can be obtained, using the single pump head curve point flow rate values together with doubled head values (see Fig. 4).

The series circuit of two pumps together with the existing pipeline provides a head of $30 \mathrm{~m}\left(D_{S}\right.$ in Fig. 4) instead of the intended $36 \mathrm{~m}$. The parallel circuit of two pumps together with the existing pipeline provides flow rate only $1.751 \mathrm{~s}^{-1}\left(D_{P}\right.$ in Fig. 4$)$ instead of the intended $3.21 \mathrm{~s}^{-1}$. 


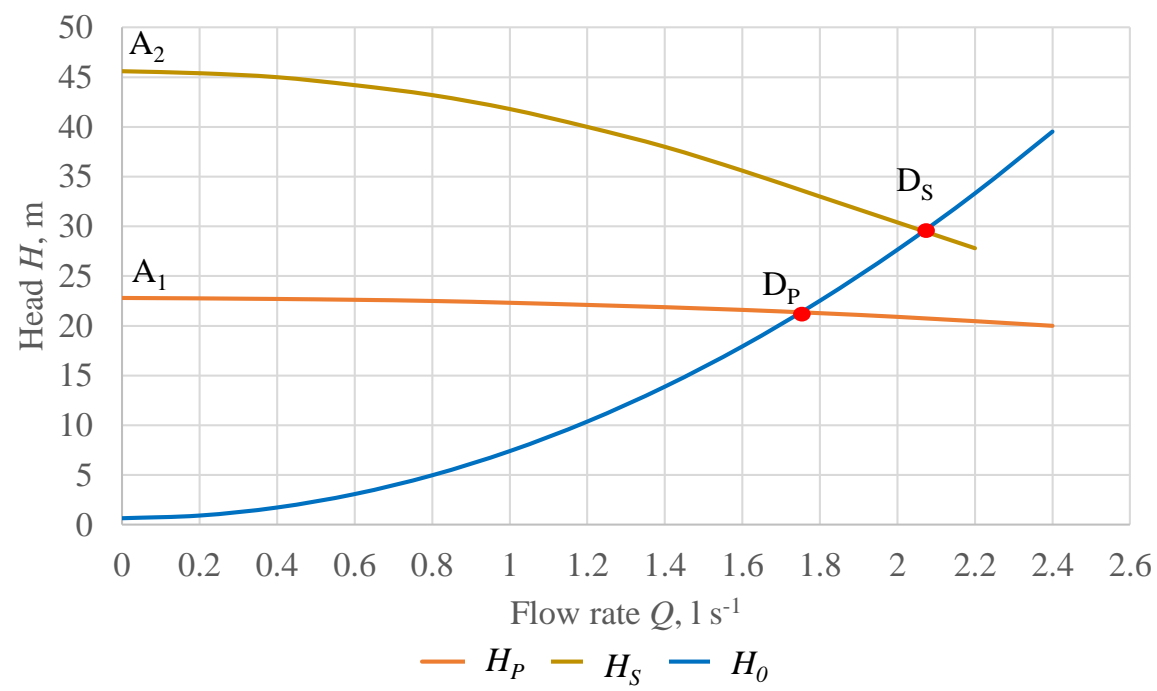

Fig. 4. Parallel and series application of two pumps

Displaying a graphic of double centrifugal pump series and parallel application main head characteristics together with the pipeline network characteristic student is gaining confidence that double centrifugal pumps together do not provide double flow rate nor either double head.

\section{Conclusions}

1. The pump head value difference of experimentally obtained characteristic and that, described by parabolic equation, is less than $5.7 \%$ for $Q=0.81 \cdot \mathrm{s}^{-1}$.

2. The analytical parabolic main characteristic of the pump and also different individual parabolic equations of the pipeline network equation (2) for each student let obtain understanding of the method of adjusting the pump parameters.

3. Two pump parallel application can be obtained, using the single pump head curve point head values together with doubled flow rate values.

4. Two pump series application can be obtained, using the single pump head curve point flow rate values together with doubled head values.

5. Displaying a graphic of double centrifugal pump series and parallel application main head characteristics together with the pipeline network characteristic is gaining confidence that double centrifugal pumps together do not provide double flow rate nor either double head.

\section{References}

[1] Gevorkov L. Model Based Power Estimation for Centrifugal Pumps. Conference Paper, 16th International Symposium "Topical Problems in the Field of Electrical and Power Engineering", January 17, 2017, 5 p.

[2] Si Q., Bois G., Liao M., Zhang H., Cui Q., Yuan S. A Comparative Study on Centrifugal Pump Designs and Two-Phase Flow Characteristic under Inlet Gas Entrainment Conditions. Energies 2020, 13, $65 \mathrm{p}$.

[3] Jilani A., Razali A. Centrifugal Pump Performance Characteristics for Domestic Application. MATEC Web of Conferences 225, 2018, $6 \mathrm{p}$.

[4] Muhammad R. A. M., Washik I. M. and Khan M. H. Experimental study on performance characteristics of a small centrifugal pump. International Journal of Advance Agricultural Research. 6 (2018) 1-17. ISSN 2053-1265.

[5] Grapsas V., Stamatelos F., Anagnostopoulos J. and Papantonis D. Numerical Study and Optimal Blade Design of a Centrifugal Pump by Evolutionary Algorithms. Conference Paper. September 2008. DOI: 10.1007/978-3-540-85565-1_4. 
[6] Stan M., Pana I., Minescu M, etc. Centrifugal Pump Monitoring and Determination of Pump Characteristic Curves Using Experimental and Analytical Solutions. Processes 2018, 6, 18; doi:10.3390/pr6020018.

[7] Derakhshan S., Nourbakhsh A. Experimental study of characteristic curves of centrifugal pumps working as turbines in different specific speeds. Experimental Thermal and Fluid Science. Volume 32, Issue 3, 2008, pp 800-807.

[8] ShankarV.K.A., Subramaniam U., ElavarasanR. M., Rajue K., Shanmugam P. Sensorless parameter estimation of VFD based cascade centrifugal pumping system using automatic pump curve adaption method. Energy Reports. Volume 7, 2021, pp. 453-466.

[9] Tavares T.S. Performance Testing of Small Water Pumps: A Versatile and Economical Laboratory Exercise for Engineering Technology Students. American Society for Engineering Education, 2017. Paper ID \#19055. 